\title{
Characterization of a Novel Hemoglobin-Glutathione Adduct That Is Elevated in Diabetic Patients
}

\author{
Yousef Al-Abed, ${ }^{1}$ Sonya VanPatten, ${ }^{1}$ Hongwei Li, ${ }^{2}$ John A. Lawson, ${ }^{2}$ Garret A. FitzGerald, ${ }^{2}$ \\ Kirk R. Manogue, ${ }^{1}$ and Richard Bucala ${ }^{1}$ \\ ${ }^{1}$ The Picower Institute for Medical Research, Manhasset, New York, USA \\ ${ }^{2}$ University of Pennsylvania, Philadelphia, Pennsylvania, USA \\ Accepted June 15, 2001
}

\begin{abstract}
Background: Typically, a diagnosis of diabetes mellitus is based on elevated circulating blood glucose levels. In an attempt to discover additional markers for the disease and predictors of prognosis, we undertook the characterization of $\mathrm{HbA}_{1 \mathrm{~d} 3}$ in diabetic and normal patients.

Material and Methods: PolyCAT A cation exchange chromatography and liquid chromatography-mass spectroscopy was utilized to separate the $\alpha$ - and $\beta$-globin chains of $\mathrm{HbA}_{1 \mathrm{~d} 3}$ and characterize their presence in normal and diabetic patients.

Results: We report the characterization of $\mathrm{HbA}_{1 \mathrm{~d} 3}$ as a glutathionylated, minor hemoglobin subfraction that occurs in higher levels in diabetic patients $(2.26 \pm 0.29 \%)$ than in

normal individuals $(1.21 \pm 0.14 \%, p<0.001)$. The $\alpha$-chain spectrum displayed a molecular ion of $m / z 15126 \mathrm{Da}$, which is consistent with the predicted native mass of the $\mathrm{HbA}_{0}$ $\alpha$-globin chain. By contrast, the mass spectrum of the $\beta$-chain showed a mass excess of $307 \mathrm{Da}(\mathrm{m} / \mathrm{z}=16173 \mathrm{Da})$ versus that of the native $\mathrm{HbA}_{0} \beta$-globin chain $(\mathrm{m} / z=15866$ $\mathrm{Da})$. The native molecular weight of the modified $\beta$-globin chain $\mathrm{HbA}_{0}$ was regenerated by treatment of $\mathrm{HbA}_{1 \mathrm{~d} 3}$ with dithiothreitol, consistent with a glutathionylated adduct. Conclusions: We propose that $\mathrm{HbA}_{1 \mathrm{~d} 3}$ (HbSSG) forms normally in vivo, and may provide a useful marker of oxidative stress in diabetes mellitus and potentially other pathologic situations.
\end{abstract}

\section{Introduction}

Once a mammalian red cell is released into the blood stream, it loses its capacity to synthesize protein. During a red cell's 120-day life span, its proteins are susceptible to several posttranslational modifications, including nonenzymatic glycation and oxidation. The accumulation of such protein modifications is considered to be typical of the cellular and molecular changes associated with the aging process (1-4). Accordingly, an important clinical marker of diabetes mellitus is the hemoglobin species known as $\mathrm{HbA}_{1 \mathrm{c}}$. $\mathrm{HbA}_{1 \mathrm{c}}$ occurs when the amino terminal valine residue of the $\beta$-chain of globin becomes covalently derivatized with an Amadori product via the Maillard reaction $(5,6)$. Because the formation of the slowly reversible Amadori product depends on circulating glucose levels, this marker accumulates to a higher degree in the red cells of those diabetics with higher hyperglycemia. Similarly, the accumulation of advanced glycation endproducts (AGEs) on hemoglobin and other proteins has been used as a long-term marker of glucose control in diabetes. Using AGEs as a marker, rather than $\mathrm{HbA}_{1 \mathrm{c}}$, has the advantage of presenting an indication of circulating glucose levels over an extended period of time (1-4,7-10).

Address correspondence and reprint requests to: Yousef Al-Abed, PhD, The Picower Institute for Medical Research, 350 Community Drive, Manhasset, New York 11030. E-mail: yal-abed@picower.edu
Although no de novo protein synthesis takes place in erythrocytes, the cells exhibit very active enzymatic synthesis of reduced glutathione (GSH), which is present at an intracellular concentration of $2.3 \mathrm{mM}$. In the red cell, GSH is an essential component for the maintenance of $\mathrm{HbA}_{0}$ in a physiologically active form. Glutathione disulfide (GSSG), the oxidized form of GSH (present at a concentration of $4.0 \mu \mathrm{M})$ is either continuously reduced by the glutathione reductase system or actively transported out of the erythrocyte so as to maintain a high intracellular GSH/GSSG ratio. It has been widely reported that diabetics have lower intracellular levels of GSH, and this is considered to be indicative of increased oxidative stress in these patients (11-14). Reduced GSH levels are believed to be a result of at least three circumstances within the cell: (1) a decrease in the activity of $\gamma$-glutamyl-cysteine synthetase (the enzyme that is responsible for the first step in glutathione synthesis), possibly due to nonenzymatic glycation of the enzyme (15); (2) a decrease in the activity of glutathione reductase, the enzyme that catalyzes the reduction of GSSG to GSH (also a result of enzyme glycation) (16); and (3) a decrease in the activity of the $\mathrm{Mg}^{+2}$ ATPase transporter responsible for GSSG export resulting in an increase of the intraerythrocytic level of GSSG (13). These factors result in decreased synthesis of new glutathione, as well as decreased regeneration of GSH from GSSG, and decreased transport of GSSG to the outside of 
the red cell. This creates an ideal environment for the formation of hemoglobin (and other protein)glutathione adducts (HbSSG).

HbSSG can be synthetically prepared by reacting GSSG (17-19) with human hemoglobin in vitro. Successful experimental formation of HbSSG in vitro led to the suggestion that HbSSG might also form under in vivo conditions, but this has been shown only to occur under extraordinary circumstances of long-term therapy with red-ox active drugs. In a population of patients treated with the anti-epileptic agents phenobarbital and caramazepin, Niketic et al. (20) detected by isoelectric focusing a minor hemoglobin, designated $\mathrm{HbA}_{1 \mathrm{x}}$, which they propose to be a glutathione-modified form.

Although a simple diagnosis of "diabetes mellitus" can be made on the basis of abnormally elevated blood sugar levels, additional markers may be valuable in the diagnosis and management of the disease and in the formation of a prognosis of the development of multi-organ complications (3). Herein, we report the isolation and molecular characterization of a variant of hemoglobin, termed HbSSG $\left(\mathrm{HbA}_{1 \mathrm{~d} 3}\right)$, which occurs at higher levels in Type I diabetics than in normal subjects. Its formation from GSSG suggests that this novel hemoglobin species may represent a specific marker of oxidative stress.

\section{Materials and Methods}

Hemolysate Preparation

Human diabetic whole blood, preserved in EDTA, was generously provided by Dr. Helen Vlassara (Mount Sinai School of Medicine, NY, NY, USA). Control blood was obtained from age-matched, nondiabetic volunteers. Blood samples were centrifuged at $1800 \times \mathrm{g}$ for $10 \mathrm{~min}$ at $4^{\circ} \mathrm{C}$ to obtain packed red blood

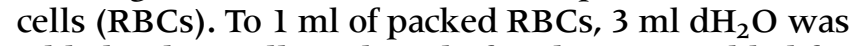
added to lyse cells and $2 \mathrm{ml}$ of toulene was added for delipidation. Vigorous vortexing for $3 \mathrm{~min}$ ensured complete extraction of lipids. Hemolysates then were centrifuged at $1800 \times \mathrm{g}$ for $10 \mathrm{~min}$ at $4^{\circ} \mathrm{C}$ to separate the aqueous from the nonaqueous phases. The nonaqueous phase was removed by aspiration and a glass pipette was used to remove the hemolysate. Hemolysates were stored at $-70^{\circ} \mathrm{C}$ until use.

\section{Fractionation of Hemoglobin Species}

To achieve separation of the various glycated hemoglobin species, PolyCAT A (PolyLC, Columbia, MD, USA) cation exchange chromatography $(200 \times$ $4.6 \mathrm{~mm}$ ) was employed. The chromatographic system consisted of a Waters $600 \mathrm{E}$ controller, a $60 \mathrm{~F}$ pump, a 996 photodiode array detector, and a 717 plus auto sampler. Buffer A was $35 \mathrm{mM}$ Bis-Tris (all chemicals in buffers purchased from Sigma, St. Louis, MO, USA), $16.85 \mathrm{mM}$ ammonium acetate, $90 \mathrm{mM}$ sodium acetate, and $1.5 \mathrm{mM}$ potassium cyanide, and was buffered to a $\mathrm{pH}$ of 6.8 with acetic acid. Buffer B was 35 mM Bis-Tris, $3 \mathrm{mM}$ ammonium acetate, and $1.5 \mathrm{mM}$ potassium cyanide, and was buffered to $\mathrm{pH} 6.5$ with acetic acid. The gradient started isocratically at $22 \% \mathrm{~A}, 78 \% \mathrm{~B}$ for the first $3 \mathrm{~min}$ and was maintained at a flow rate of $3.0 \mathrm{ml} / \mathrm{min}$ throughout the run. From 3-30 min, buffer A increased linearly to $50 \%$, and then linearly to $100 \%$ from 3040 min. Buffer A was maintained at $100 \%$ until $40.5 \mathrm{~min}$ and then decreased to $22 \%$ over the next $5 \mathrm{~min}$. The column then was equilibrated over the next 20 min with the initial solvent ratio. Fractions were collected upon monitoring absorbance at $415 \mathrm{~nm}$.

\section{LC-MS Methods}

$\mathrm{A} \mathrm{C}_{4}$-reversed phase column $(1 \times 50 \mathrm{~mm})$ (Vyadec, $5 \mu \mathrm{M})$ at a flow rate of $50 \mu \lambda / \mathrm{min}$ was used for $\alpha$ - and $\beta$-chain separation. Solvent C contained $0.05 \%$ triflouroacetic acid (TFA) in $\mathrm{ddH}_{2} \mathrm{O}$, and solvent D contained $0.05 \%$ TFA in acetonitrile. The column was eluted with a binary C:D solvent gradient beginning at $40 \% \mathrm{D}$ and linearly increasing to $60 \% \mathrm{D}$ in $20 \mathrm{~min}$. Column eluate was monitored at $214 \mathrm{~nm}$, and peaks were analyzed by electrospray ionization mass spectrometry (combined LC-ESI) wherein ESI spectra were scanned from 10,00-35,000 mass units at a scan cycle of $5 \mathrm{sec} / \mathrm{scan}$.

\section{Results}

\section{Isolation of Human Hemoglobin}

Figure 1 illustrates the fractionation scheme we used to isolate the various human hemoglobin species in this study. Whole blood hemolysates were obtained from 20 patients with a wide spectrum of severity and duration of diabetes. Results of PolyCAT cation exchange chromatographic fractionation of the hemolysate of one representative patient is illustrated in Figure 2. The $\mathrm{HbA}_{1 \mathrm{c}}$ peak was found to represent $14.0 \%$ of total hemoglobin and was confirmed by LC-MS (21). As indicated in

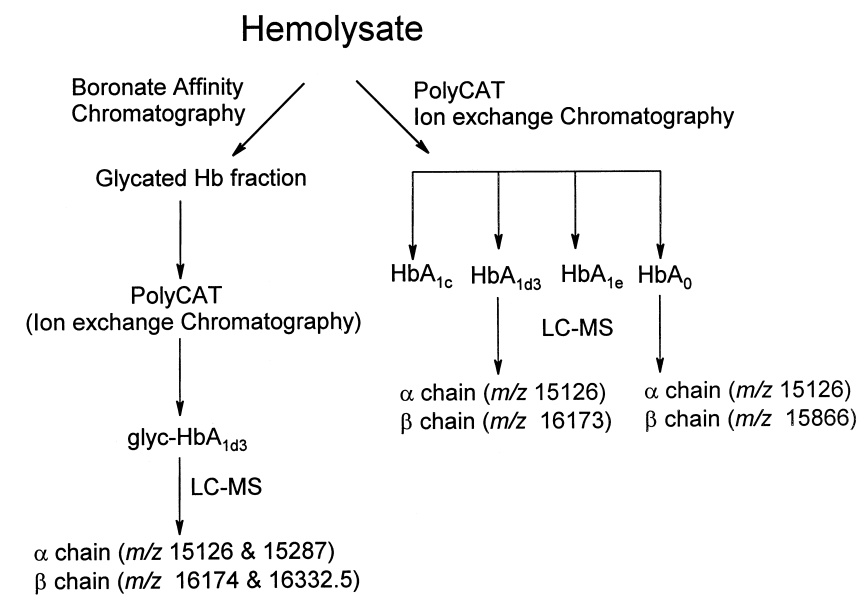

Fig. 1. Schematic diagram for the isolation of different hemoglobin extracts separated by PolyCAT and LC-MS. 

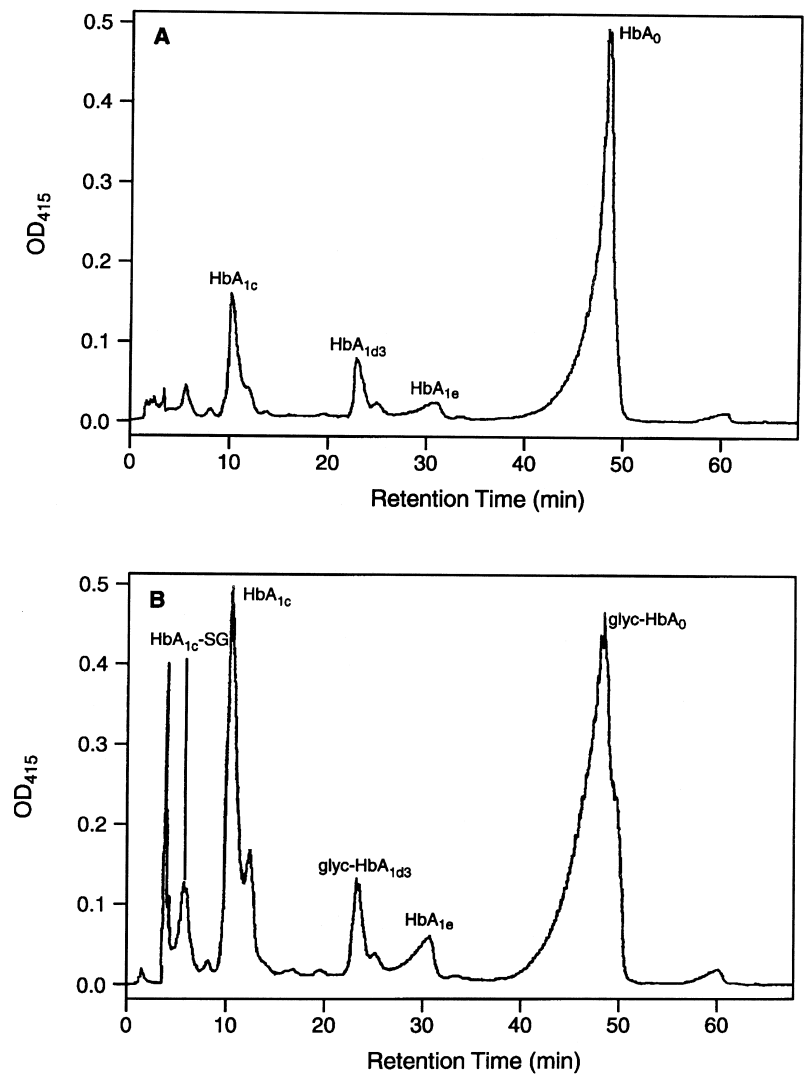

Fig. 2. Cation exchange and boronate affinity chromatography analyses of human diabetic hemoglobin. (A) Elution of hemoglobin peaks from a delipidated whole blood hemolysate obtained from a human diabetic patient and fractionated by cation exchange chromatography. Hemoglobin subfractions were assigned by identification of constituent globin chains as determined by subsequent HPLC and LCMS analyses. HbA, hemoglobin with no modification to either the $\alpha$ - or $\beta$-chains; $\mathrm{HbA}_{1 \mathrm{c}}$, hemoglobin with an Amadori glycation product attached to the amino terminal valine of the globin $\beta$-chain; $\mathrm{HbA}_{1 \mathrm{~d} 3}$, hemoglobin modified by attachment of oxidized glutathione (HbASSG) as a mixed disulfide with the cysteine side chain at position 93 of the globin $\beta$-chain; $\mathrm{Hb}_{1 \mathrm{e}}$, hemoglobin with an Amadori glycation product attached to the amino terminal valine of the globin $\alpha$-chain. (B) Fractionation of glycated hemoglobin fraction obtained by boronate affinity chromatography by ion exchange chromatography. glyc-HbA $\mathrm{A}_{0}$, hemoglobin that is glycated at the lysine side chain of either the $\alpha$ - or $\beta$-chains (Al-Abed et al. unpublished data); $\mathrm{HbA}_{1 \mathrm{c}}-\mathrm{SG}$, hemoglobin with a simultaneous Amadori glycation product and glutathionylation at cysteine side chain at position 93; glyc- $\mathrm{HbA}_{1 \mathrm{~d} 3}$, glycated hemoglobin that is also modified by attachment of glutathione (HbASSG) to the cysteine side chain at position 93 of the globin $\beta$-chain; $\mathrm{HbA}_{1 \mathrm{e}}$, hemoglobin with an Amadori glycation product attached to the amino terminal valine of the globin $\alpha$-chain.

Figure 2A, the minor hemoglobin species conventionally have been designated according to their elution order. The $\mathrm{HbA}_{1 \mathrm{~d} 3}$ subfraction contained $2.26 \pm 0.29 \%$ of total hemoglobin in the samples. By comparison with previously published chromatograms (21), the identity of this subfraction was expected to represent $\mathrm{HbA}_{0}$ in which globin had become modified either by glutathione or a glycation product.
Separation and Characterization of the $\alpha$ - and $\beta$-Globin Chains of $\mathrm{HbA}_{1 \mathrm{~d} 3}$

Liquid chromatography-mass spectrometry (LC-MS) was applied to separate the $\alpha$ - and $\beta$-globin chains of $\mathrm{HbA}_{1 \mathrm{~d} 3}$ (Fig. 3A). The $\alpha$-chain spectrum displayed a molecular ion of $\mathrm{m} / \mathrm{z} 15126 \mathrm{Da}$, which is consistent with the predicted mass of native $\mathrm{HbA}_{0}$ $\alpha$-globin chain (Fig. 3B). By contrast, the mass spectrum of the $\beta$-chain showed a mass excess of $307 \mathrm{Da}$ versus that of the native $\mathrm{HbA} \mathrm{HbA}_{0} \beta$-globin chain (Fig. 3C). To establish that this modification was due to glutathione $(m / z 307 \mathrm{Da})$, the $\mathrm{HbA}_{1 \mathrm{~d} 3}$ was incubated with DTT in phosphate-buffered saline (PBS) under $\mathrm{N}_{2}$ for $24 \mathrm{hr}$ and then analyzed by ion exchange chromatography and LC-MS. The ion exchange elution profile indicated that the reduced $\mathrm{HbA}_{1 \mathrm{~d} 3}$ was eluted in a similarly to native $\mathrm{HbA}_{0}$. Moreover, the mass spectrum of the $\beta$-chain from DTT-reduced $\mathrm{HbA}_{1 \mathrm{~d} 3}$ was identical to that of $\mathrm{HbA}_{0}$. The $\beta$-chain of hemoglobin contains two cysteinyl
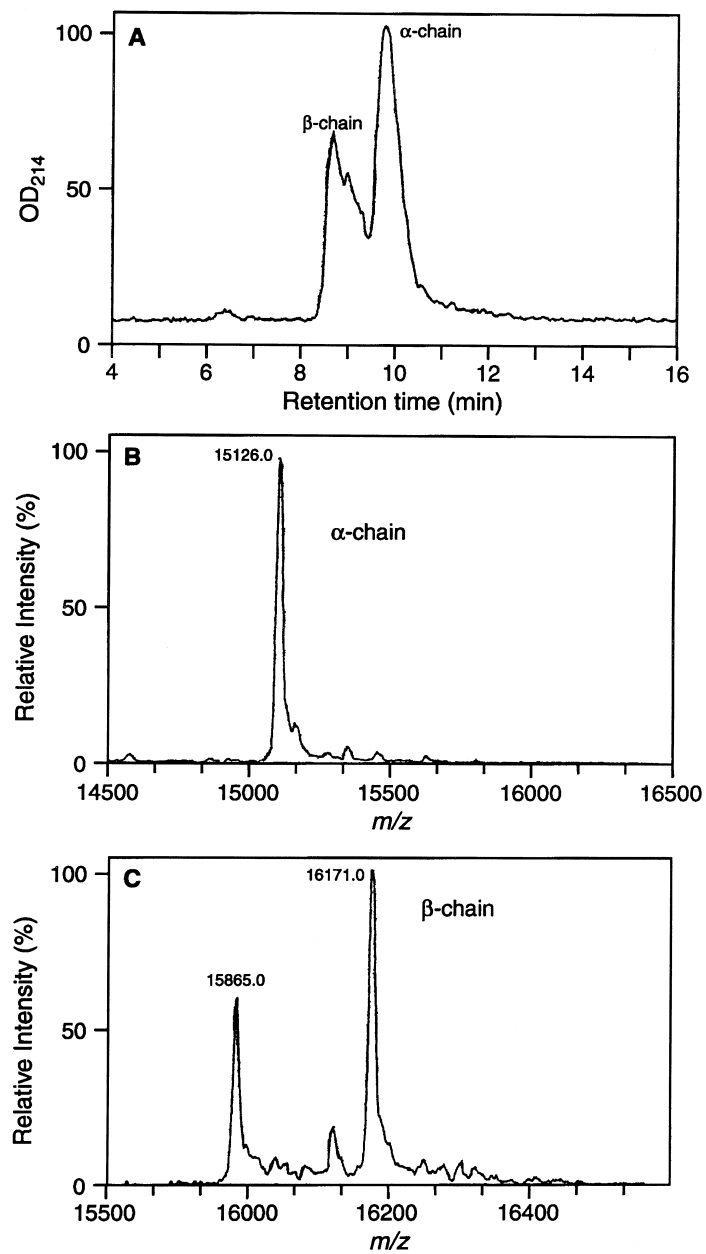

Fig. 3. LC-MS analyses of $\mathrm{HbA}_{1 \mathrm{~d} 3}$ (HbSSG). (A) Fractionation of hemoglobin subfraction $\mathrm{HbA}_{1 \mathrm{~d} 3}$ into component globin chains by $\mathrm{C}_{4}$-reversed phase HPLC. (B) Mass spectrum of globin $\alpha$-chain. (C) Mass spectrum of globin $\beta$-chain. 
residues at position $\beta-93$ and $\beta-112$, and the $\alpha$ subunit has one cysteinyl residue at position $\alpha-104$. The $\beta-112$ and $\alpha-104$ residues are internally oriented and are involved in subunit contact, but do not form a disulfide bridge, making them generally unavailable for modification. Taken together, these data are consistent with the modification of the $\beta$-globin Cys ${ }^{93}$ by oxidized gluthatione. (The expected mass for the $\beta$-chain of $\mathrm{HbA}_{0}$ is 15,866 plus 307 for covalent glutathione adduct $=16,172$, versus the measured $\mathrm{HbA}_{1 \mathrm{~d} 3} m / z=16,173[\mathrm{M}+\mathrm{H}]^{+}$.)

\section{Susceptibility of Glycated $\mathrm{Hb}$ to Glutathione Modification}

To determine whether glycated fractions of the same diabetic hemolysates are susceptible to glutathione modification, hemolysates were subjected to boronate affinity chromatography, which selectively retains glycated $\mathrm{Hb}$ species. Then, the bound fractions then were eluted and further fractionated using ion exchange chromatography (Fig. 1B). The fraction corresponding to glycated $\mathrm{HbA}_{1 \mathrm{~d} 3}$ (glyc$\left.\mathrm{HbA}_{1 \mathrm{~d} 3}\right)$ was isolated and subjected to LC-MS. Data presented in Figure 4 indicate the molecular weight of each chain in this glyc-HbA $\mathrm{Ad}_{3}$ subfraction. As expected, the mass of the $\beta$-chain was consistent with simultaneous covalent glutathionylation and glycation. Mass spectrometry analysis of this fraction after DTT treatment revealed a mass ion consistent only with glycation modification (mass excess = 162), the glutathione adduct having been removed by reduction (data not shown). We also detected glycated species that are glutathionylated, such as the

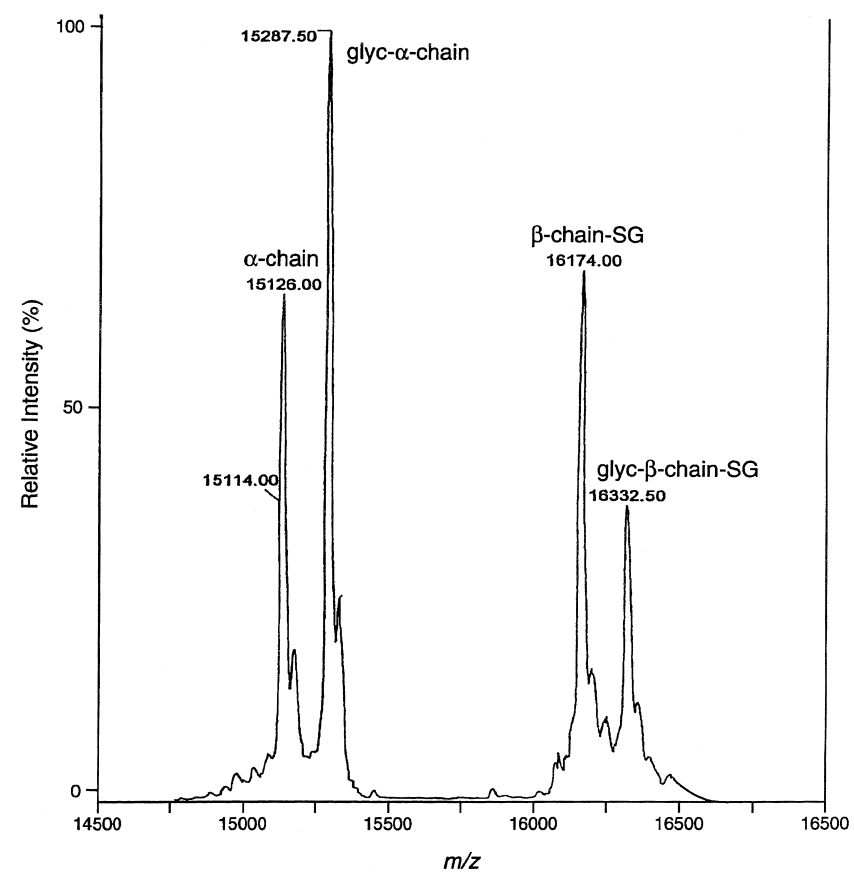

Fig. 4. Mass spectrum of glyc-HbA $A_{1 d 3}$ subfraction obtained by ion exchange chromatography of the glycated hemoglobin as shown in Figure $1 B$. glutathionylated $\mathrm{HbA}_{1 \mathrm{c}}\left(\mathrm{HbA}_{1 \mathrm{c}}-\mathrm{SG}\right)$ shown in Figure 2B. On the basis of LC-MS and analysis, it is apparent that multiple hemoglobin variants such as $\mathrm{HbA}_{0}$, $\mathrm{HbA}_{1 \mathrm{c}}$, and $\mathrm{HbA}_{1 \mathrm{~d} 3}$, are susceptible to glutathionylation, indicating that there is an underestimation of the total glutathionylated hemoglobin content by simple ion exchange chromatography.

\section{$H b A 1_{d 3}$ and $H b A_{1 d 3}$ Levels in Non-diabetic} and Diabetic Individuals

We next measured the level of $\mathrm{HbA}_{1 \mathrm{~d} 3}$ in nine healthy volunteers and compared the values with those from diabetic subjects. Hemolysates were prepared and fractionated by ion chromatography as above. $\mathrm{HbA}_{1 \mathrm{c}}$ was found to be $2.2 \pm 0.77 \%$ of total hemoglobin, and its identity (glycated at $\mathrm{NH}_{2}$-terminal valine of $\beta$-globin chain) was confirmed by LC-MS (data not shown). The $\mathrm{HbA}_{1 \mathrm{~d} 3}$ peak was quantified and found to represent $1.21 \pm 0.14 \%$ of total $\mathrm{Hb}$. These values were significantly different from those of diabetic patients, whose $\mathrm{HbA}_{1 \mathrm{c}}$ levels were $9.30 \pm 2.49 \%$ of total hemoglobin, and whose $\mathrm{HbA}_{1 \mathrm{~d} 3}$ levels were $2.26 \pm 0.29 \%$ of total hemoglobin (Fig. 5A).The levels of gluthionylated $\mathrm{Hb}$ adducts in diabetic patients correlated wit the level of $\mathrm{HbA}_{1 \mathrm{c}}$ as shown in Figure 5B. Moreover, the level of $\mathrm{HbA}_{1 \mathrm{~d} 3}$ in diabetic and normal individuals was comparable to the levels of the newly characterized glycated hemoglobin $\mathrm{HbA}_{1 \mathrm{e}}$, (Figs. 2AB) in which an Amadori glycation product is attached to the amino terminal valine of the globin $\alpha$-chain (Al-Abed et al., in preparation).

\section{Discussion}

We previously identified a glutathionylated hemoglobin in diabetic rats using ion exchange chromatography and LC-MS $(21)$. Niwa et al. $(22,23)$ recently reported the identification of a glutathionylated $\mathrm{HbA}_{0}$ that is present at higher levels in diabetic (Type I) and hyperlipidemic patients. It was concluded in that study that there is no correlation between the formation of $\mathrm{HbA}_{1 \mathrm{c}}$ and $\mathrm{HbA}_{1 \mathrm{~d} 3}$ in diabetic and hyperlipidemic patients. Their finding was based however on detecting a mass unit of 16,173 Da for a modified $\beta$-globin subfraction that corresponds to glutathionylated $\beta$-globin and runs parallel with the native $\beta$-globin chain in reverse phase HPLC. The elevated level of this glutathionylated $\mathrm{Hb}$ in diabetic (Type I) and hyperlipidemic patients was based on a nonquantitative mass spectra analysis.

In summary, we have isolated and characterized $\mathrm{HbA}_{1 \mathrm{~d} 3}$, a normally occurring minor hemoglobin subfraction that results from a glutathione adduct at the $\beta$-93 cysteine residue (HbSSG). This adduct accounts for $1.21 \pm 0.14 \%$ versus $2.26 \pm 0.29 \%$ of the total hemoglobin in normal individuals and diabetic patients, respectively. We propose that the minor hemoglobin species $\mathrm{HbA}_{1 \mathrm{~d} 3}$, or HbSSG, forms normally in vivo, and may provide a useful marker of oxidative stress in a variety of pathologic situations. 
A

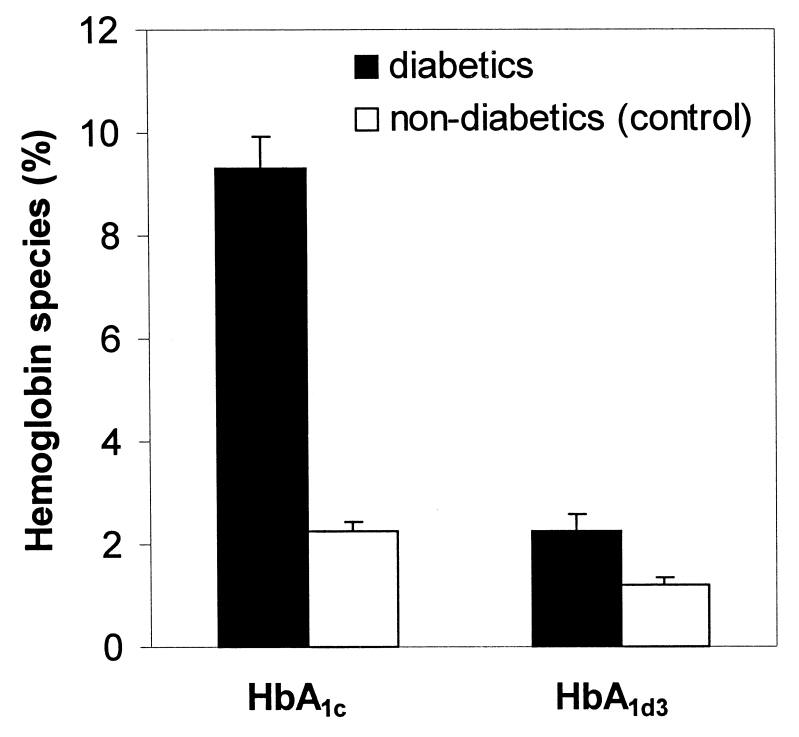

B

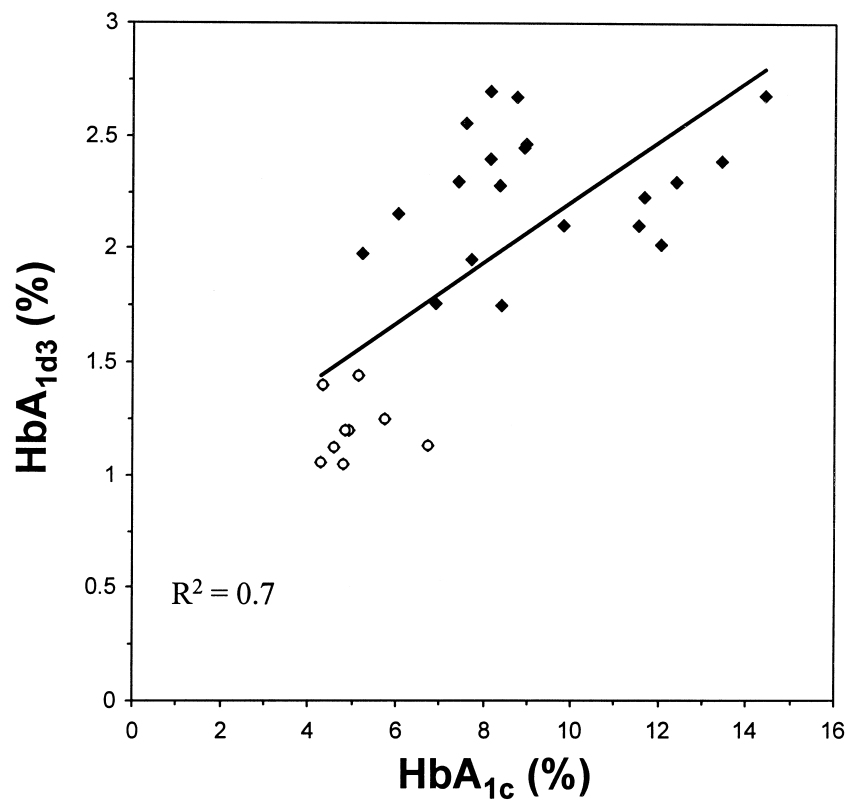

Fig. 5. $\mathrm{HbA}_{1 \mathrm{~d} 3}$ and $\mathrm{HbA}_{1 \mathrm{c}}$ levels. (A) Comparison of concentrations of $\mathrm{HbA}_{1 \mathrm{~d} 3}$ and $\mathrm{HbA}_{\mathrm{Ic}}$ in diabetic $(n=20)$ and non-diabetic individuals $(n=9), p<0.001$ for diabetic versus nondiabetic $\mathrm{HbA}_{\mathrm{Ic}}$ and $p<0.001$ for diabetic versus nondiabetic $\mathrm{Hb}_{1 \mathrm{~d} 3}$. (B) Correlation plot between $\mathrm{HbA}_{1 \mathrm{~d} 3}$ and $\mathrm{HbA}_{\mathrm{Ic}}$ in diabetic $(\bullet)$ and nondiabetic $(\diamond)$ individuals, $y=0.1341 \mathrm{x}+0.8634$.

\section{References}

1. Dryer DG, Dunn JA, Thorpe SR, et al. (1993). Accumulation of Maillard reaction products in skin collagen in diabetes and aging. J. Clin. Invest. 91: 2463-2469.

2. Ledl F, Schleicher E. (1990). The Maillard reaction in food and in human body-New results in chemistry, biochemistry and medicine. Angew. Chem. Int. Ed. Engl. 6: 565-594.

3. Brownlee M, Cerami A, Vlassara H. (1998). Advanced glycosylation end products in tissue and the biochemical basis of diabetic complications. N. Engl. J. Med. 318: 1315- 1321.

4. Bucala R, Cerami A. (1992). Advanced glycosylation: chemistry, biology, and implications for diabetes and aging. Adv. Pharmacol. 23: 1-34.

5. Rahbar S. (1968). Hemoglobin $H$ disease in two Iranian families: Clin. Chim. Acta. 22: 296-299.

6. Koenig RJ, Blobstein SH, Cerami A. (1977). Structure of carbohydrate of hemoglobin AIc. J. Biol. Chem. 252: 29922997.

7. Makita Z, Vlassara H, Cerami A, Bucala R. (1992). Immunochemical detection of advanced glycosylation end products in vivo. J. Biol. Chem. 267: 5133-5138.

8. Schleicher E, Wagner E, Nerlich AG. (1997). Increased accumulation of the glycoxidation product $\mathrm{N}$ (epsilon)(carboxymethyl)lysine in human tissues in diabetes and aging. J. Clin. Invest. 99: 457-468.

9. Makita Z, Vlassara H, Rayfield E, et al. (1992). HemoglobinAGE: a circulating marker of advanced glycosylation. Science 258: 651-653.

10. Wolffenbuttel BH, Giordano D, Founds HW, Bucala R: (1996). Long-term assessment of glucose control by hemoglobinAGE measurement. Lancet 347: 513-515.

11. Gandhi CR, Roy Chowdhury RD. (1979). Effect of diabetes mellitus on sialic acid $\delta$ glutathione content of human erythrocytes of different ages. Indian J. Exp. Biol. 17: 585-587.

12. Jain SK, McVie R. (1994). Effect of glycemic control, race (white versus black), and duration of diabetes on reduced glutathione content in erythrocytes of diabetic patients. Metabolism 43: 306-309.

13. Ciuchi E, Odetti P, Prando R. (1996). Relationship between glutathione and sorbitol concentrations in erythrocytes from diabetic patients: Metabolism 45: 611-613.

14. Murakami K, Kondo T, Ohtsuka Y, Fujiwara Y, Shimada M, Kawakami Y. (1989). Impairment of glutathione metabolism in erythrocytes from patients with diabetes mellitus. Metabolism 38: $753-758$.

15. Murakami K. (1991). Glutathione metabolism in erythrocytes from patients with diabetes mellitus. Hokkaido Igaku Zasshi 66: 29-40.

16. Blakytny R, Harding JJ. (1992). Glycation (non-enzymic glycosylation) inactivates glutathione reductase. Biochem $J$ 288: 303-307.

17. Birchmeier W, Tuchschmid PE, Winterhalter KH. (1973). Comparison of human hemoglobin A carrying glutathione as a mixed disulfide with the naturally occurring human hemoglobin A. Biochemistry 12: 3667-3672.

18. Wodak SJ, De Coen JL, Edelstein SJ, Demarne H, Beuzard Y. (1986). Modification of human hemoglobin by glutathione. III. Perturbations of hemoglobin conformation analyzed by computer modeling. J. Biol. Chem. 261: 14717- 14724.

19. Garel MC, Domenget C, Caburi-Martin J, Prehu C, Galacteros F, Beuzard Y. (1986). Covalent binding of glutathione to hemoglobin. I. Inhibition of hemoglobin S polymerization. J. Biol. Chem. 261: 14707-14724.

20. Niketic V, Beslo D, Raicevic S, Sredic S, Stojkovic M. (1992). Glutathione adduct of hemoglobin (Hb ASSG) in hemolysates of patients on long-term antiepileptic therapy. Int. J. Biochem. 24: 503-507.

21. Al-Abed Y, Mitsuhashi T, Li H, et al. (1999). Inhibition of advanced glycation endproduct formation by acetaldehyde: role in the cardioprotective effect of ethanol. Proc. Natl. Acad. Sci. U.S.A. 96: 2385-2390.

22. Niwa T, Naito C, Mawjood AHM, Imai K. (2000). Increased glutathionyl hemoglobin in diabetes mellitus and hyperlipidemia demonstrated by liquid chromatography/electrospray ionization-mass spectrometry. Clin. Chem. 46: 82-88.

23. Naito C, Kajita M, Niwa TJ. (1999). Determination of glutathionyl hemoglobin in hemodialysis patients using electrospray ionization liquid chromatography-mass spectrometry. Chromatogr. B 731: 121-124. 